\title{
Safety and Efficacy of MLC601 in Iranian Patients after Stroke: A Double-Blind, Placebo-Controlled Clinical Trial
}

\author{
A. A. Harandi, ${ }^{1,2}$ R. Abolfazli, ${ }^{3}$ A. Hatemian, ${ }^{4}$ K. Ghragozlee, ${ }^{1}$ M. Ghaffar-Pour, ${ }^{3}$ \\ M. Karimi, ${ }^{5}$ S. Shahbegi, ${ }^{5}$ H. Pakdaman, ${ }^{1}$ M. Tabasi, ${ }^{6}$ A. L. Tabatabae, ${ }^{3}$ and A. Nourian ${ }^{7}$ \\ ${ }^{1}$ Shaheed Beheshti University of Medical Sciences and Health Services, Tehran 19839-63113, Iran \\ ${ }^{2}$ Department of Neurology, Loghman Hospital, Shahid Beheshti University of Medical Sciences, Tehran 13336-31151, Iran \\ ${ }^{3}$ Tehran University of Medical Sciences and Health Services, Tehran, Iran \\ ${ }^{4}$ Gylan University of Medical Sciences and Health Services, Gylan, Iran \\ ${ }^{5}$ Milad Hospital, Tehran, Iran \\ ${ }^{6}$ Social Security, Tehran, Iran \\ ${ }^{7}$ Azad University of Medical Sciences, Tehran 19168, Iran
}

Correspondence should be addressed to A. A. Harandi, amini_alli@yahoo.com

Received 7 October 2010; Revised 22 February 2011; Accepted 26 April 2011

Academic Editor: Daniel Bereczki

Copyright () 2011 A. A. Harandi et al. This is an open access article distributed under the Creative Commons Attribution License, which permits unrestricted use, distribution, and reproduction in any medium, provided the original work is properly cited.

Objective. To investigate the safety and efficacy of MLC601 (NeuroAid) as a traditional Chinese medicine on motor recovery after ischemic stroke. Methods. This study was a double-blind, placebo-controlled clinical trial on 150 patients with a recent (less than 1 month) ischemic stroke. All patients were given either MLC601 (100 patients) or placebo (50 patients), 4 capsules 3 times a day, as an add-on to standard stroke treatment for 3 months. Results. Sex, age, elapsed time from stroke onset, and risk factors in the treatment group were not significantly different from placebo group at baseline $(P>.05)$. Repeated measures analysis showed that Fugl-Meyer assessment was significantly higher in the treatment group during 12 weeks after stroke $(P<.001)$. Good tolerability to treatment was shown, and adverse events were mild and transient. Conclusion. MLC601 showed better motor recovery than placebo and was safe on top of standard ischemic stroke medications especially in the severe and moderate cases.

\section{Introduction}

Stroke is one of the major leading causes of death and disability worldwide [1]. Thrombolytic therapies in selected patients and antiplatelet prescription have been remained as just two main approved medications in this setting. Standard treatment modalities in stroke rehabilitation are limited to physiotherapy, occupational therapy, and speech therapy in addition to skilled medical and nursing care. Despite intensive inpatient rehabilitation with these modalities in stroke units, about $1 / 3$ of acute stroke patients remained moderately to severely disabled at discharge time. Many studies tried to find new therapeutic strategies to improve outcome of treatment in stroke; however, results of several clinical trials on variety of neuroprotective drugs have been failed to show efficacy in humans [2].
Some promising combination therapies have been introduced by traditional Chinese medicine (TCM). It consists of several types of medicinal herbs, based on more than 2500 years of clinical experience. MLC601 (NeuroAid) is a TCM which is used extensively in China to improve recovery after stroke [3]. It combines several herbal and animal components. Some studies evaluated its safety and efficacy. In addition, the neuroprotective and/or neurogenerative properties in vitro and in vivo assays have been assessed. However, further studies are needed to approve the different aspects of effectiveness on mental and poststroke motor function and also to clear its pharmacokinetics. For prescription of MLC601 as a standard drug in stroke, more documents especially in human field should be provided to improve decision making based on evidence-based medicine. For this reason, we decided to investigate the safety and 
efficacy of MLC601 on motor recovery in ischemic stroke patients.

\section{Methods}

2.1. Study Design and Subjects. In a double-blind, placebocontrolled clinical study, the safety and efficacy of NeuroAid (Moleac Pte. Ltd, Singapore) were compared with controls in ischemic stroke. The study protocol was registered in http://ClinicalTrials.gov/ (Identifier: NCT01336153). The period of the recruitment was from July 2009 to February 2010. The subjects were recruited from in- and out-clinic patients from Iran. Blocked randomization was used to randomly allocate 100 and 50 participants to MLC601 and placebo group, respectively. Block sizes of 2 and 3 and an allocation ratio of $2: 1$ (e.g., ABABAA and ABABAAABA) were applied in witch A was twofold more than $B$. The study profile is supplied as Figure 1.

Both drug and placebo were similarly packaged and marked as A or B, respectively. All subjects were allocated to either group A (100 patients; NeuroAid 400 mg, 4 capsules 3 times daily) or group B (50 patients; placebo, 4 capsules 3 times daily) and received placebo or NeuroAid for 3 months. MLC601 (0.4 g per capsule) combines 9 herbal (0.57g Radix astragali, 0.114 g Radix salvia miltiorrhizae, 0.114 g Radix paeoniae rubra, 0.114 g Rhizoma chuanxiong, $0.114 \mathrm{~g}$ Radix angelicae sinensis, $0.114 \mathrm{~g}$ Prunus persica, $0.114 \mathrm{~g}$ Carthamus tinctorius, $0.114 \mathrm{~g}$ Radix polygalae, and $0.114 \mathrm{~g}$ Rhizoma acori tatarinowii,) and 5 animal components (0.0285 g Cornu saigae tataricae, $0.095 \mathrm{~g}$ Buthus martensii, 0.0665 Hirudo, $0.0665 \mathrm{~g}$ Eupolyphaga seu steleophaga, and $0.0285 \mathrm{~g}$ Calculus bovis artifactus). In order to prepare the placebo, similar capsules in shape and color were filled with flour as inert chemical structure, thus making them indistinguishable from the original capsules. The patients were visited at the beginning and every 10 days in the first month and every two weeks in the second and third months. NeuroAid or placebo was added to the standard regimen of patients including antiplatelet, anticoagulant, lipid lowering agents, antihypertensive, and antidiabetic, medications. Treatment was initiated less than 4 weeks after stroke. All patients underwent neurological evaluation, computerized tomography (CT), magnetic resonance imaging (MRI) of brain, electrocardiogram, blood investigation, full blood count, renal function test, liver function test, electrolytes, prothrombin time (PT), and partial thromboplastin time (PTT) at first and monthly.

Inclusion criteria were age between 30-72 years, less than one month after stroke, ischemic cerebral stroke, and signed informed consent form. Exclusion criteria were treatment with thrombolytic, ischemic stroke combined with hemorrhage, severe renal or liver failure, dementia, psychosis and history of seizure disorder, history of previous stroke, and hemoglobin level less than $10 \mathrm{mg} / \mathrm{dL}$ on admission.

2.2. Outcome Measure. The efficacy endpoint was improvement in affected upper and lower limbs impairment as assessed by the Fugl-Meyer assessment (FMA) scale at baseline, 4, 8, and 12 weeks. Patients were categorized into 3

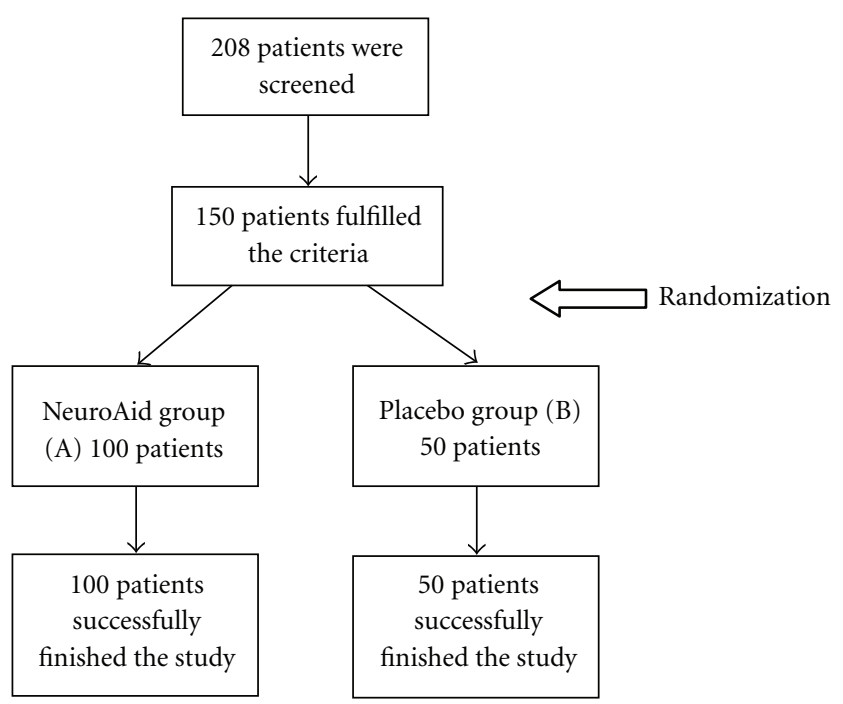

FIgUre 1: The study profile.

categories according to their baseline FMA score at initiation of the clinical trial: severe (0-55), moderate (56-80), and mild (81-100).

The data were analyzed with independent $t$-test and repeated measured analysis using SPSS version 11.0 software (SPSS Inc., Chicago, Ill, USA). $P$ values $\leq .05$ were considered statistically significant.

2.3. Ethical Issues. The study was conducted in accordance with the principles embodied in the declaration of Helsinki, and the project design was approved by an appropriate institutional ethics committee. All patients or in cases with severe disability their first relatives had signed an informed consent form.

\section{Results}

Patients were neither excluded nor withdrawn or lost to followup. The NeuroAid and the control group had similar baseline characteristics in gender, age, elapsed time from stroke onset, and stroke severity (Table 1 ). Table 2 shows the FMA scores comparison between the NeuroAid and the placebo groups at four different points in time, that is, at baseline, 4, 8, and 12 weeks after stroke. By generalized linear model, repeated measured analysis was performed which showed a statistically significant difference in trend of FMA in two groups during 12 weeks of follow up $(P<.0001$; Figure 2).

Safety. There were no severe side effects which led to discontinuation of NeuroAid. The common side effects were mild and transient nausea and vomiting in 7 patients who took NeuroAid. There were no abnormal changes in blood count or renal and liver function.

\section{Discussion}

Our results cleared that considering FMA, patients who received MLC601 showed significantly better motor recovery 
TABLE 1: Baseline characteristics of two groups.

\begin{tabular}{lccc}
\hline Group & $\begin{array}{c}\text { NeuroAid } \\
(N=100)\end{array}$ & $\begin{array}{c}\text { Placebo } \\
(N=50)\end{array}$ & $P$ value \\
\hline Sex (male) $N(\%)$ & $48(48 \%)$ & $24(52 \%)$ & .99 \\
Age (mean \pm SD) & $64.41 \pm 5.89$ & $66.14 \pm 5.36$ & .08 \\
Risk factors & & & .48 \\
$\quad$ Hypertension & 56 & 31 & .90 \\
$\quad$ Diabetic mellitus & 43 & 22 & .64 \\
$\quad$ Hyperlipidemia & 48 & 22 & .99 \\
$\quad$ Ischemic heart disease & 11 & 3 & .99 \\
$\quad$ Smoking & & 5 & .53 \\
Stroke details & $9.45 \pm 5.39$ & $8.92 \pm 3.51$ & .90 \\
Days since stroke & & & \\
Site of the hemiparesis & 47 & 24 & 26 \\
$\quad$ Right & 53 & & \\
$\quad$ Left & &
\end{tabular}

Table 2: Fugl-Meyer Assessment score at baseline and after treatment in the NeuroAid and the placebo group.

\begin{tabular}{lccc}
\hline FMA & NeuroAid $($ no. $=100)$ & Placebo $($ no. $=50)$ & $P$ value \\
\hline Baseline & $53.69 \pm 23.01$ & $54.96 \pm 24.27$ & .755 \\
4 weeks after intervention & $77.13 \pm 19.22$ & $63.50 \pm 24.21$ & $<.001^{*}$ \\
8 weeks after intervention & $82.51 \pm 14.27$ & $72.06 \pm 21.41$ & $.001^{*}$ \\
12 weeks after intervention & $86.22 \pm 12.34$ & $74.36 \pm 18.1$ & $<.001^{*}$ \\
\hline
\end{tabular}

FMA: Fugl-Meyer assessment. * Statistically significant.

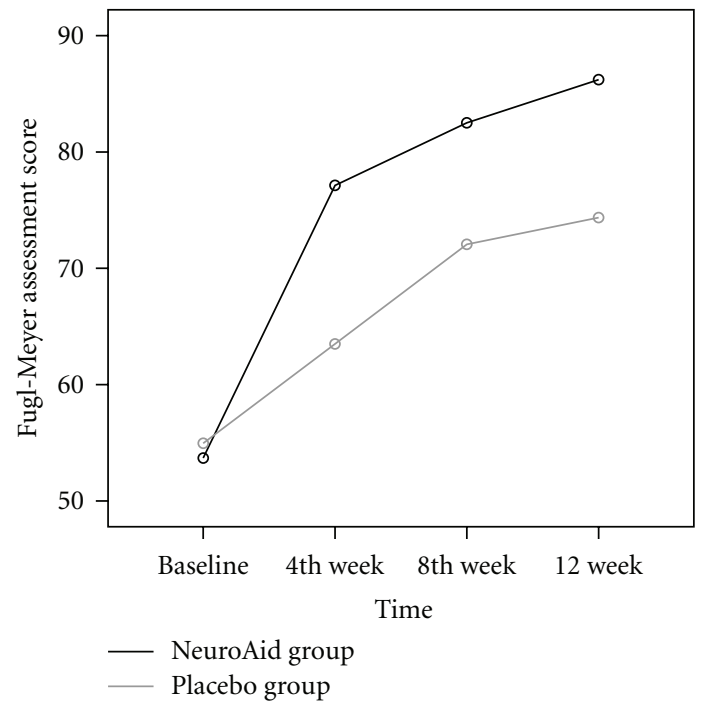

Figure 2: FMA scores in two groups at baseline, 4,8 , and 12th week (repeated measure analysis). $P$ value for groups $=.003 ; P$ value for time $<.0001 ; P$ value for interaction between groups and time for 3 times after intervention $=.439$.

than placebo group after 4,8 , and 12 weeks. The improvement was mostly manifested after first month and developed more during 12 weeks. In addition, no considerable side effects were recorded. In this study, we focused on motor function to obtain more specific and reliable data. Our findings provide a new evidence to support efficacy and tolerability of MLC601 in post ischemic stroke patients.

Clinical researches based on TCM have provided new treatments for cerebral infarction. More than 100 TCM agents have been approved by Chinese National Drug Administration and are used clinically in China for stroke [4]. It was developed to aid post stroke recovery and was approved in 12 countries including Iran. Pharmacological studies demonstrated some TCMs to have antioxidant, antiinflammatory, and antiglutamate effects [5]. However, these have limited acceptability by Western medicine because of lack of available evidences for the efficacy and safety of TCM [3]. A recent meta-analysis of TCM for ischemic stroke only found clinical trial reports for 59 TCM and concluded that the methodological quality of most trials were poor. According to this report, only 3 studies were randomized, double-blind, and placebo-controlled, in which 2 had longterm outcome assessments [4]. It is clear that further large, well-designed trials are necessary.

Early trials of MLC601 were performed in China on 605 patients in 2000, established its safety, and demonstrated a positive effect on the recovery of independence and motor functions. The patients were found to be 2.4 times more likely to achieve independence at 1 month after stroke than the control group $[3,6,7]$. Our results showed the same tendency for better recovery in the first month.

Two similar randomized clinical trials comparing the efficacy and safety of NeuroAid and BNJ (a TCM approved by the Sino Food and Drug Administration) in subjects with recent ischemic stroke were included in a pooled analysis. 
Two hundred subjects were randomized in the first study and 405 in the second. Most of separate motor function pooled analyses showed an advantage in those subjects randomized to the MLC601 treatment group compared with the control group. Specifically, MLC601 decreased the scores at 1 month for the 2 domains of upper limb and distal lower limbs as compared with the active control. A numeric decrease in the scores for lower limb and facial and distal upper limb functions was shown without statistically significant differences. No significant effect was observed on visual and language functions. Drug was well tolerated in two trials [3].

In a double-blind, placebo-controlled pilot study by Kong et al. that was more similar to our study, the efficacy of NeuroAid on motor recovery in ischemic stroke patients was assessed using rehabilitation endpoints. Forty subjects less than one month postischemic stroke were given either NeuroAid or placebo, 4 capsules 3 times a day for 4 weeks. FMA, National Institute of Health Stroke Scale and Functional Independence Measure scores were measured at baseline, 4 and 8 weeks. None of the outcomes were significantly different from placebo group except for FMA scores. Consistent with our study, FMA scores showed a positive trend for improvement with NeuroAid treatment over time. Posterior circulation and severe stroke subjects showed a tendency for better recovery [8].

In a case series, 10 patients received NeuroAid 4 tablets, 3 times per day between 1 week and 6 months after stroke for 2-3 months. The patients showed a good tolerability and only 1 patient reported diarrhea as a mild side effect after starting NeuroAid. During follow-up period, all cases reported improvements in which 6,3 , and 1 patients showed full, good or moderate and poor recovery, respectively. Significant improvements were recorded in motor, visual, speech, and cognitive functions [7].

In a multicenter study called Chinese Medicine MLC601 Efficacy on Stroke recovery (CHIMES), a total of 114 patients were recruited after Month 3. At the time of analysis, 13 patients were lost to followup. Therefore, only 51 patients on treatment A and 49 on treatment B had laboratory data available for this analysis. According to interim analysis, there were neither statistically or clinically significant differences between groups in biochemical, heamatological, or ECG tests nor in the absolute and relative changes in the various parameters over 3 months. This study is still ongoing [9].

In a rodent model of focal ischemia, NeuroAid (MLC601) and NeuroAid II (MLC901) showed interesting neuroprotective and neurogenesis benefits both in vivo and in vitro at different stages after stroke. MLC901 treatment, when administered in vivo in before or after stroke improved animal survival as well as functional neurological recovery and decreased neurodegeneration without affecting physiological parameters. MLC601 and MLC901 also prevented neuronal death in vivo and in vitro model of excitotoxicity and could induce neurogenesis in rodent and human cells, promote cell proliferation as well as neurite outgrowth and stimulate the development of a dense axonal and dendritic network [10]. We believe it was one of the most interesting studies with most precise results in this field.
Furthermore, it has been recently shown that NeuroAid, taken either alone or in combination with aspirin, does not change hemostasis, hematology, and biochemistry in normal subjects and stroke patients [11]. Therefore, it can be used safely with the mentioned drug. It was also well tolerated in our study.

\section{Conclusion}

Our study as a randomized, double-blind, placebocontrolled clinical trial shows significant motor recovery and NeuroAid was safe on top of standard ischemic stroke medication. However, still more clinical trials are needed to evaluate safety and efficacy of NeuroAid for stroke recovery before such treatments can be recommended for routine and standard poststroke treatment. Many questions still remained to be answered, for example, what are interactions with other drugs, when is the optimal time for initiation of therapy, how long is duration of treatment, which age groups are the best candidate, and what are the long term-benefits.

\section{Acknowledgment}

The authors would like to appreciate Farshid Alaeddini, M.D., Ph.D., Health Researchers R \& D Institute, Tehran, Iran for statistical analysis.

\section{References}

[1] C. D. Mathers and D. Loncar, "Projections of global mortality and burden of disease from 2002 to 2030," PLoS Medicine, vol. 3, no. 11, article e442, pp. 2011-2030, 2006.

[2] M. D. Ginsberg, "Neuroprotection for ischemic stroke: past, present and future," Neuropharmacology, vol. 55, no. 3, pp. 363-389, 2008.

[3] C. Chen, N. Venketasubramanian, R. N. Gan et al., "Danqi Piantang Jiaonang (DJ), a traditional Chinese medicine, in poststroke recovery," Stroke, vol. 40, no. 3, pp. 859-863, 2009.

[4] B. Wu, M. Liu, H. Liu et al., "Meta-analysis of traditional Chinese patent medicine for ischemic stroke," Stroke, vol. 38, no. 6, pp. 1973-1979, 2007.

[5] W. D. Rausch, S. Liu, G. Gille, and K. Radad, "Neuroprotective effects of ginsenosides," Acta Neurobiologiae Experimentalis, vol. 66, no. 4, pp. 369-375, 2006.

[6] Q. Tang, "Clinical study report on efficiency of danqi piantan capsule in the treatment of apoplexy due to deficiency of qi and stasis of blood syndrome," Zhong Guo Zhong Yi Yao Ke Ji, vol. 10 , p. 69, 2003.

[7] C. H. C. Siow, "Neuroaid in stroke recovery," European Neurology, vol. 60, no. 5, pp. 264-266, 2008.

[8] K. H. Kong, S. K. Wee, C. Y. Ng et al., "A doubleblind, placebo-controlled, randomized phase II pilot study to investigate the potential efficacy of the traditional Chinese medicine Neuroaid (MLC 601) in enhancing recovery after stroke (TIERS)," Cerebrovascular Diseases, vol. 28, no. 5, pp. 514-521, 2009.

[9] N. Venketasubramanian, C. L. H. Chen, R. N. Gan et al., "A double-blind, placebo-controlled, randomized, multicenter study to investigate CHInese Medicine Neuroaid Efficacy on Stroke recovery (CHIMES Study)," International Journal of Stroke, vol. 4, no. 1, pp. 54-60, 2009. 
[10] C. Heurteaux, C. Gandin, M. Borsotto et al., "Neuroprotective and neuroproliferative activities of NeuroAid (MLC601, MLC901), a Chinese medicine, in vitro and in vivo," $\mathrm{Neu}$ ropharmacology, vol. 58, no. 7, pp. 987-1001, 2010.

[11] R. Gan, C. Lambert, J. Lianting et al., "Danqi Piantan Jiaonang does not modify hemostasis, hematology, and biochemistry in normal subjects and stroke patients," Cerebrovascular Diseases, vol. 25, no. 5, pp. 450-456, 2008. 


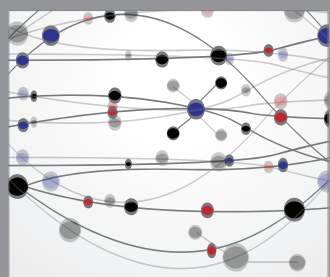

The Scientific World Journal
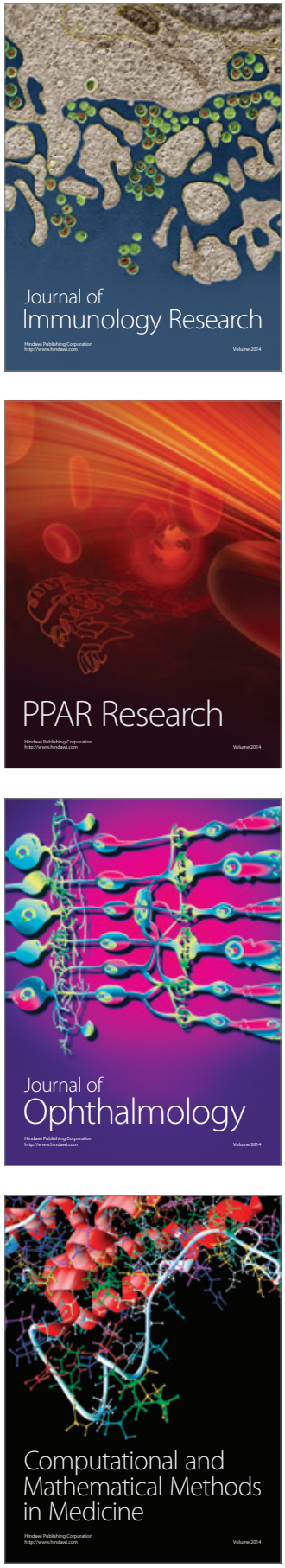

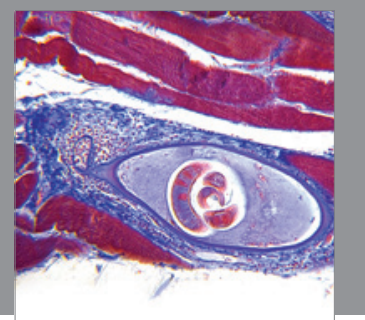

Gastroenterology

Research and Practice
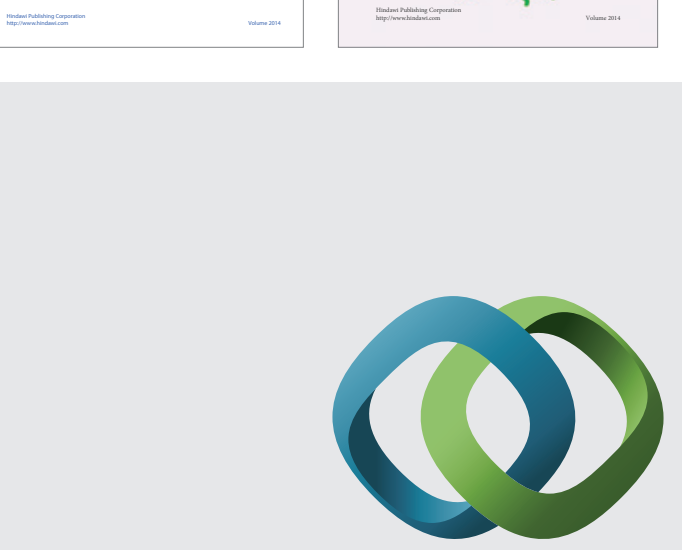

\section{Hindawi}

Submit your manuscripts at

http://www.hindawi.com
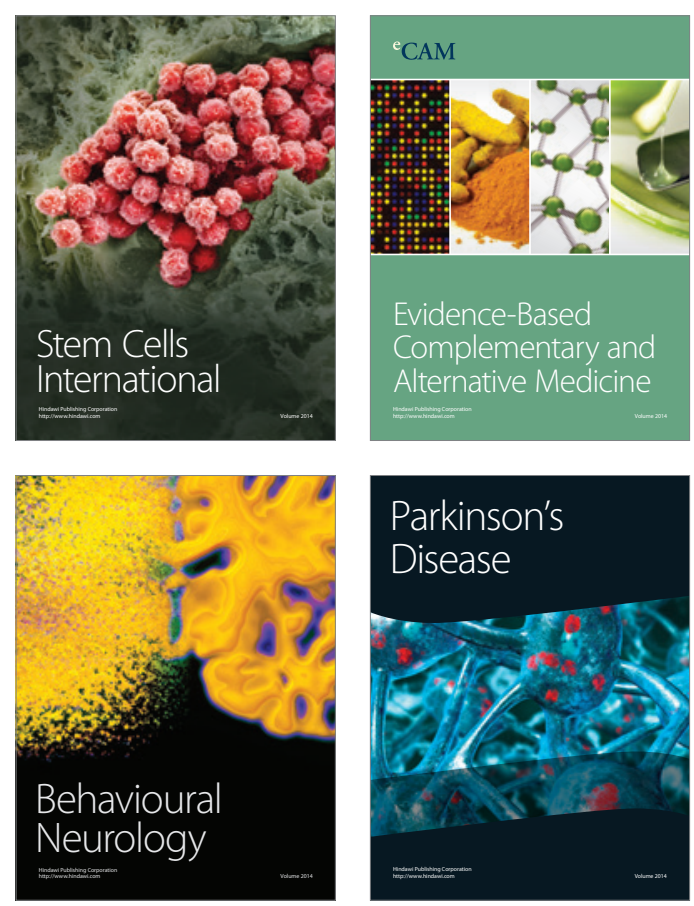

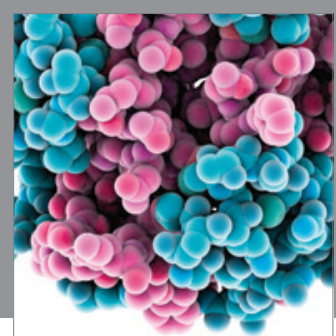

Journal of
Diabetes Research

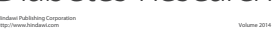

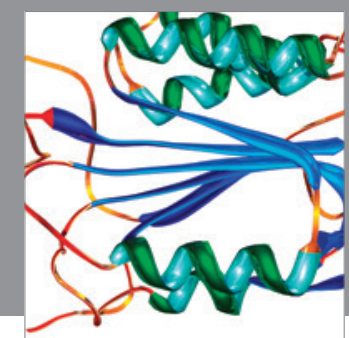

Disease Markers
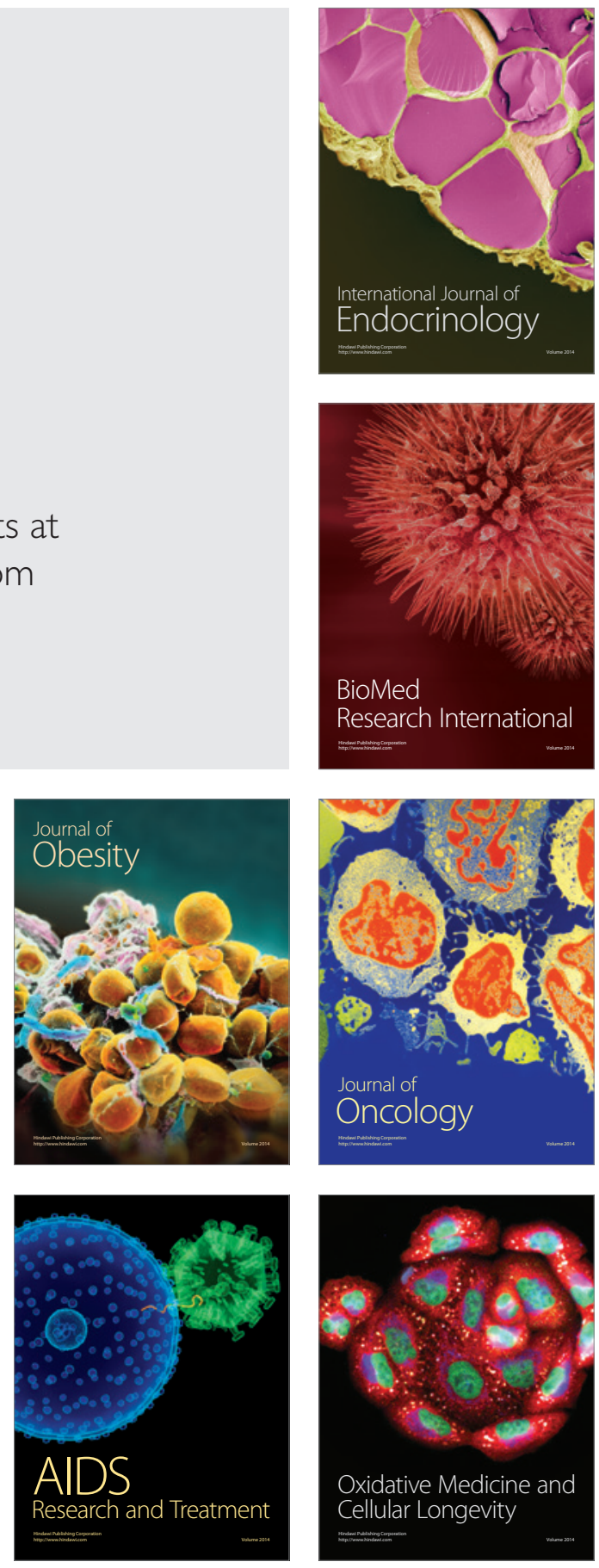